\title{
SUPERVISED MACHINE LEARNING AND OCR IMPLEMENTATION IN SYSTEM DESIGN OF NUMBER PLATE RECOGNITION AUTOMATICALLY
}

KatrojuVivek $^{1} \&$ Sumit Bhardwaj $^{2}$

Abstract-Supervised machine learning and OCR implementation in system design of Number plate Recognition Automatically is an image processing technology which uses license plates for the identification of vehicle. It is a one of the kind of Optical Character Recognition (OCR). This system is implemented in many areas for security and the identification of the owner of a particular vehicle. The use of automatic number plate recognition system has become very significant in our daily life because of increase in transportation vehicles and reduce in security for those vehicles due to theft and etc., Due to different formats and styles of number plate in this paper we mainly presented an algorithm apart from OCR TRANIER TOOL from MATlab r2016 for vehicle number identification which uses supervised machine learning, Histogram of oriented gradients (HOG) etc., The software used in this paper is MATlab r2016a.

Keywords: Optical Character Recognition (OCR); Supervised Machine Learning; Histogram Oriented Of Gradient (HOG) Automatic number plate recognition (ANPR).

\section{INTRODUCTION}

The concept of number plate detection automatically was developed in 1976 at the Police scientific Improvement Branch in the $\mathrm{UK}^{[4]}$. In any case, it increased much enthusiasm amid the most recent decade alongside the change of advanced camera what's more, the expansion in computational limit. It is basically the capacity to consequently concentrate and recognize the characters of a number plate from the vehicle taken from a given picture.

Basically it consists of a camera or frame grabber which has a ability to get a picture, identify the region of the number in the picture and then remove the characters for the recognition of characters to make an exposition of the pixels into numerically character with a meaning.

Supervised machine learning and OCR implementation in system design of Number plate Recognition Automatically is a mass inspection method which uses optical character recognition on images to read the license plates on vehicles. This method can be implemented in vast areas like red-light violation at traffic signals, custom checkpoints, traffic monitoring, tracking stolen vehicles and also by police forces in many many ways.

ANPR can be used to save the images which are taken by the camera and texts from the license plate, by configuring to save images in the disk. A high power flash is used in at least one type of the junction recording cameras, used for both to take a picture and to make the victim aware of his or her mistake. ANPR system to be specified in each place, due to plate variation from area to area, but we introduce as many as fonts in this paper so that it can be used through the world without causing as much as problem which are being faced now for recognition.

The main motive of this paper is to identify the licence number of a vehicle regardless of any type of font and size on the number plate.

Section 2 in this paper describes the propound algorithm and section 3 shows the result and finally section 4 finally wind-up this paper.

\section{PREVIOUS ALGORITHM:}

Qadri ${ }^{[4]}$ suggested a way for recognition of vehicle number plate and has shown the simulated results of identification of number plate using OCR, and has given certain limitations as follows:

- Sensitivity for recognition to misalignment and to different sizes using OCR method.

- Probability of recognition and identification of number plate for a vehicle using statistical analysis.

K.I Kim ${ }^{[6]}$ strictly developed only for identification of Korean plates. It has developed a system for implementing supervised Machine learning and submit the average identification capacity of a character.

Sunil Karamchandani ${ }^{[1]}$ has given a vehicle num. detection algorithm based on OCR using artificial neural network. This method gives high range of approximate identification rate of number plate with zero identification rate.

\footnotetext{
${ }^{1}$ Dept. Of Electronics and Communication Engg, Amity School Of Engineering, Amity University, Noida, U.P-201301.

${ }^{2}$ Dept. Of Electronics and Communication Engg, Amity School Of Engineering, Amity University, Noida, U.P-201301.
} 
$\mathrm{X} . \mathrm{Ye}^{[7]}$ given a 2 phase hybrid recognition system joining factual and basic recognition technique. This work incorporates recognizing comparative characters by neighbourhood basic components and building up a framework engineering consolidating factual and auxiliary identification techniques. In the first place, the four sub-classifiers freely perceive the character and after that identification results are joined utilizing the Bayes technique. Besides, if the perceived characters have a place with the arrangements of indistinct characters the auxiliary stage is utilized for further choice.

\section{PROPOSED ALGORITHM}

In this paper we are going to discuss about two methods for identification of number plate. The two methods are as follows:

A. Using OCR trainer application.

B. Using supervised machine learning algorithm

\subsection{Using OCR Trainer App}

In Matlab OCR trainer app instructs the ocr function to recognize a custom language or font ${ }^{[12]}$. You can use this app directly to generate number plate from a photo taken by the camera with minimum amount of effort. The only disadvantage of this app is it can only able to read a custom language or font not all kinds of font are read easily through the app.

The following image is subjected to OCR trainer app.

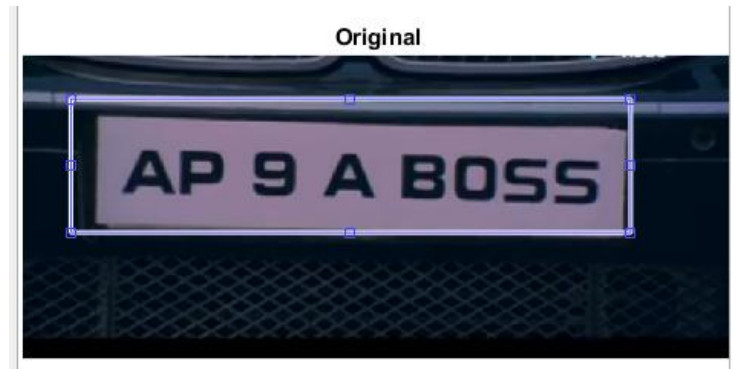

Fig 1(a). Original image of a license plate before being segmented in OCR trainer app.

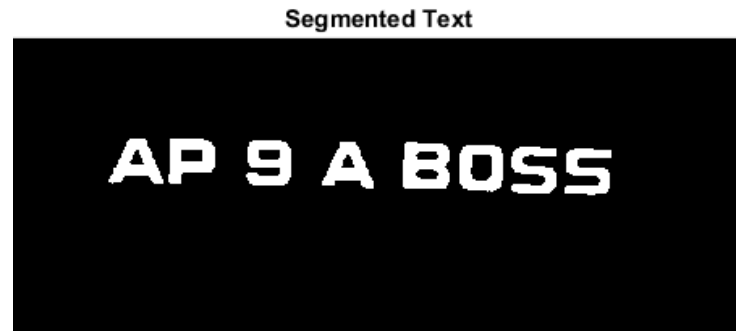

Fig 1(b). Segmented image text after being subjected to OCR trainer app.

When the image of number plate is subjected to OCR trainer app as shown in Fig 1(a) gives as an segmented image output as shown in Fig 1(b) through which we can be able to find the number plate of a vehicle directly using OCR trainer app.

\subsection{Supervised Machine Learning Algorithm}

Machine learning is a kind of artificial intelligence which gives a computer the ability to learn without being unquestionably programmed. Supervised learning is the one type of the machine learning task which conclude a function from labelled training data.

Supervised learning is used majorly in practical machine learning. In this our main goal is to approximate the mapping function so that when we have a new input data we can be able predict the output variables of that data from the database. It is of two types training and testing.

Training:

- Datasets- images(data)

- Feature extraction- features<-labelling

Testing

- Features

- Classifiers

With the help of Supervised machine learning algorithm we can get a unique property which has a pattern or boundary by which letters will be distinguished. 
With this algorithm we are using histogram of oriented gradient (HOG) and K-Nearest Neighbour classifier.

The advantage of this algorithm is it can be able to read more than 300 different fonts because we are using database of different fonts. Whereas the limitation of this algorithm is that the recognition of letters in plate are done by creating rectangle boundary for each letter in the plate, if there are more than two rectangles formed apart from the letters from the plate it couldn't able to read the given licence plate.

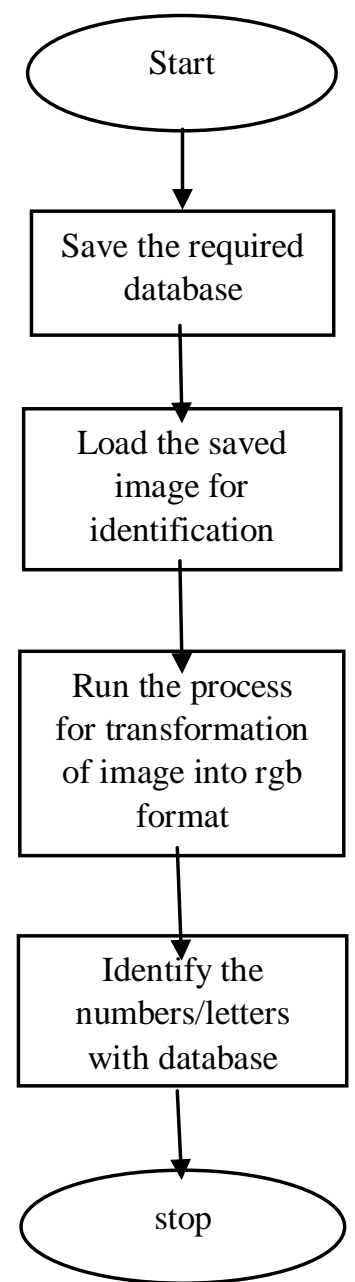

Fig 2 Proposed Algorithm

There are two types for recognition of number plate with the help of this algorithm.

i. Offline method

ii. Online processing method

\section{3, Offline Method}

In this method we upload an image from our data which we have taken already with the help of camera and find out the registered licence plate of the vehicle as shown in Fig 2(a).

\subsection{Online Processing Method}

The main difference in these two methods is that we give an input image in offline method whereas in this we take a picture of a licence plate and process it directly in real time as shown in Fig 2(b). 


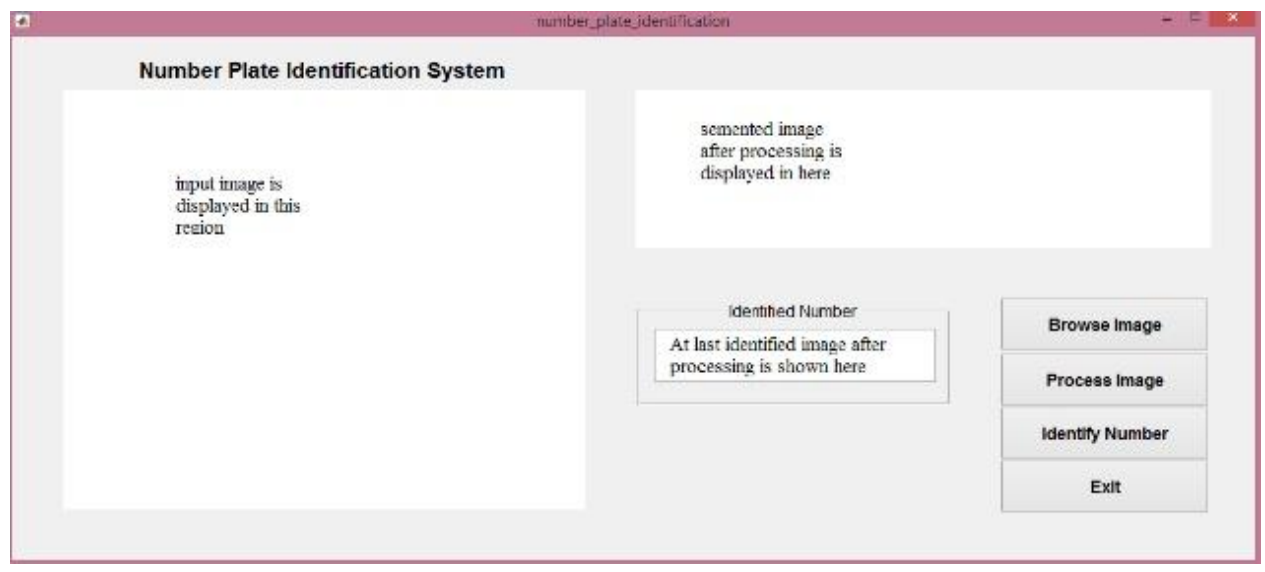

Fig 3(a). Offline processing method

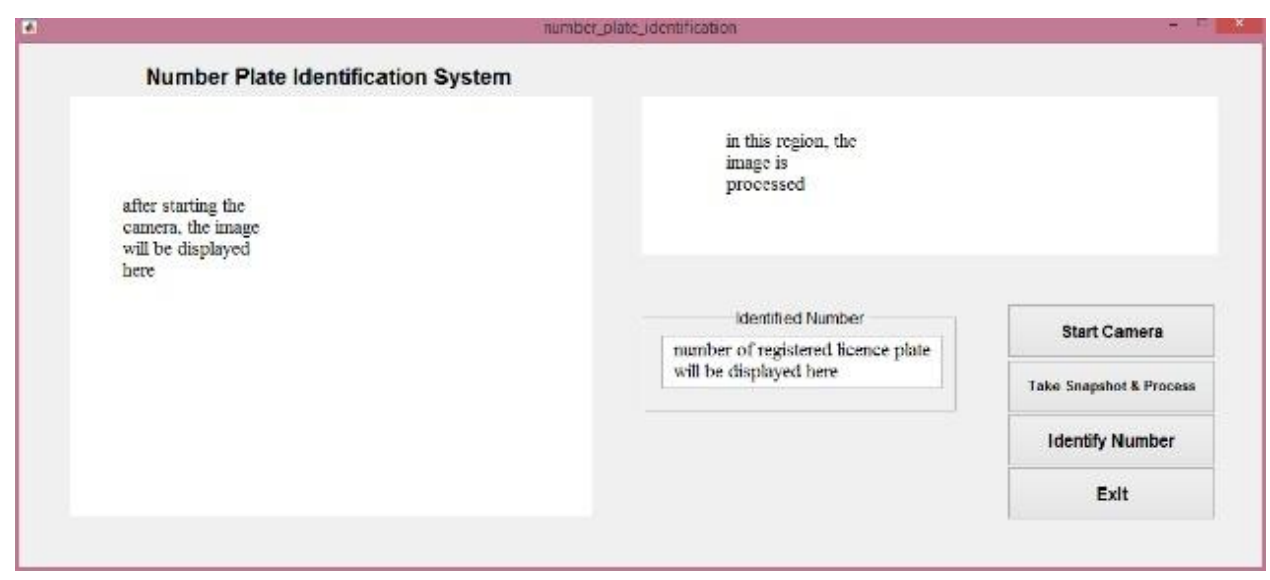

Fig 3(b). Online processing method

\subsection{Results}

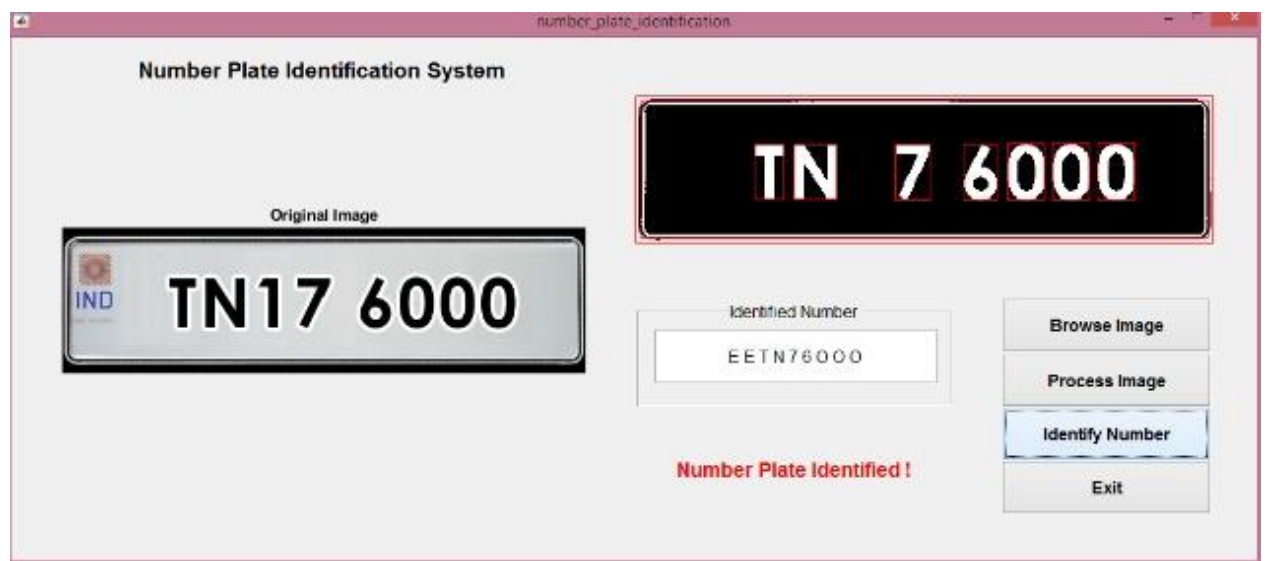

Fig 4 number plate identification system offline method

The figure 4 shows the result of the identification of the licence plate when an image is uploaded and processed.

Number identification can be done in two ways as we discussed earlier.

Firstly, we can take picture with help of an camera and upload it in Fig 3(a) so that we will be getting an output image by identifying the number plate of a given vehicle as shown in Fig 4, and another method is to take image with the help of camera and process it directly as shown in Fig 3(b). 


\section{CONCLUSION}

In this paper we conclude and evaluate the perfection of OCR process and the proposed algorithm and we have found some limitations in the proposed work i.e., If more number of rectangular boundaries are formed during the recognition of plate, it makes difficult to identify the licence plate and there is a certain probability in miscalculation of letters from each other. ANPR is tested on snapshots of static vehicles, and if an image is blurry then the chance of recognition of number plate be reduced.

\section{REFERENCES}

[1] Sunil Karamchandani, Kaushik Koli, Ketan Lad, Taskeen Nadkar, Nikita Gulechha, Parul Shah, "OCR-based Chassis-Number Recognition using Artificial Neural Networks", presented at the IEEE ICVES 2009, pp. 31-34.

[2] ,I.E.Anagnostopoulos, I.D. Psoroulas, E.KayafasLicense and V. Loumos, C.N.E.Anagnostopoulos Plate Recognition From Still Images and Video Sequences: A Survey, IEEE Transactionson Intelligent Transportation Systems 9 (2008), no. 3, 377-391.

[3] S. H. Yu, Y. S. Chen. and J. W. Hsieh Morphology based licenseplate detection from complex scenes. 16th International Conferenceon Pattern Recognition (ICPR'02), pp. 79-179, 2002.

[4] Asif ,Tahir Qadri, anpr system for vehicle identification using OCR,2009 IEEE,DOI 10.1109/ICETC.2009 pp .335-338

[5] P. Federl and J.R. Parker an Approach To Licence Plate Recognition, University of Calgary 1996.

[6] K. I. Kim, K. K. Kim H. J. Kim AND J. B. Kim, "Learning-based approach for license plate recognition", in Proc. IEEE Signal Process. Soc. Workshop, Neural Network Signal Process. 2000, vol. 2, pp. 614-623.

[7] X. Ye, X. Pan and S. Zhang, "A hybrid method for robustcar plate character recognition", presented at the IEEE International Conference on Systems, Man and Cybernetics, 2004, pp. 4733-4737.

[8] S. Ganesan and V. Kasmat, "An efficient implementation of the Hough transform for detecting vehicle license plates using DSP's," IEEE RTCSA, Chicago, USA, p.p. 58/59, 2005

[9] K. Jung, S.H. Park, H.J. Kim and K.I. kim , "Locating car license plate using Neural Network,” Electronic Letters, Vol. 35, No. 17, pp. 1474- 1477, 1999.

[10] S. Alaverdyan, G. Petrosyan and H. Sarukhanyan, number plate detection automatically,in the 7th ICCSIT, Yerevan, Armenia, September 28/2 Oct. 2009, Electronic Copy of the CSIT 2009 Proceedings, 347-350.

[11] G. Gluhchev, V. Velichkov, V. Shapiro D. Dimov and S. Bonchev, Adaptive License Plate Image Extraction, in fifth ICCSIT Rousse, Bulgaria, June 17/18, 2004 (K. Boyanov), ACM New York, NY, USA (2004), 1/7.

[12] Ahmed M Thabet, Amr Badar, Mohamed M Abdelwahab, Ahmed M Abdelsadek, Annals of the University of Craiova, Mathematics and Computer Science Series Volume 38(1), 2011, Pages 62-71.ISSN: 1223-6934 